\section{Natural history of Waldenström macroglobulinemia following acquired resistance to ibrutinib monotherapy}

\author{
Joshua N. Gustine, ${ }^{1,2}$ Shayna Sarosiek, ${ }^{1,3}$ Catherine A. Flynn, ${ }^{1}$ Kirsten Meid, ${ }^{1}$ \\ Carly Leventoff, ${ }^{1}$ Timothy White, ${ }^{1}$ Maria Luisa Guerrera, ${ }^{1}$ Lian Xu, ${ }^{1}$ \\ Amanda Kofides, ${ }^{1}$ Nicholas Tsakmaklis, ${ }^{1}$ Manit Munshi, ${ }^{1}$ Maria Demos, ${ }^{1}$ \\ Christopher J. Patterson, ${ }^{1}$ Xia Liu, ${ }^{1}$ Guang Yang, ${ }^{1,3}$ Zachary R. Hunter, ${ }^{1,3}$ \\ Andrew R. Branagan, ${ }^{3,4}$ Steven P. Treon ${ }^{1,3}$ and Jorge J. Castillo ${ }^{1,3}$ \\ ${ }^{1}$ Bing Center for Waldenström's Macroglobulinemia, Dana-Farber Cancer Institute; \\ ${ }^{2}$ Boston University School of Medicine; ${ }^{3}$ Department of Medicine, Harvard Medical \\ School and ${ }^{4}$ Division of Medical Oncology, Massachusetts General Hospital, Boston, \\ MA, USA
}

\section{ABSTRACT}

T brutinib is highly active and produces long-term responses in patients with Waldenström macroglobulinemia (WM), but acquired resistance can occur with prolonged treatment. We therefore evaluated the natural history and treatment outcomes in $51 \mathrm{WM}$ patients with acquired resistance to ibrutinib monotherapy. The median time between ibrutinib initiation and discontinuation was 2 years (range, 0.4-6.5 years). Following discontinuation of ibrutinib, a rapid increase in serum immunoglobulin $M$ level was observed in 60\% (29/48) of evaluable patients, of whom ten acutely developed symptomatic hyperviscosity. Forty-eight patients (94\%) received salvage therapy after ibrutinib. The median time to salvage therapy after ibrutinib cessation was 18 days (95\% confidence interval [CI]: 13-27). The overall and major response rates to salvage therapy were $56 \%$ and $44 \%$, respectively, and the median duration of response was 48 months (95\% CI: 34-not reached). Quadruple-class (rituximab, alkylator, proteasome inhibitor, ibrutinib) exposed disease (odds ratio [OR] 0.20, 95\% CI: 0.05-0.73) and salvage therapy $\leq 7$ days after discontinuing ibrutinib (OR 4.12, 95\% CI: 1.0718.9) were identified as independent predictors of a response to salvage therapy. The 5-year overall survival (OS) following discontinuation of ibrutinib was $44 \%$ (95\% CI: 26-75). Response to salvage therapy was associated with better OS after ibrutinib (hazard ratio $0.08,95 \% \mathrm{CI}$ : 0.02-0.38). TP53 mutations were associated with shorter OS, while acquired BTK C481S mutations had no impact. Our findings reveal that continuation of ibrutinib until subsequent treatment is associated with improved disease control and clinical outcomes.

\section{Introduction}

Waldenström macroglobulinemia (WM) is an immunglobulin $\mathrm{M}$ (IgM)-secreting lymphoplasmacytic lymphoma. ${ }^{1}$ Whole-genome sequencing has identified highly recurrent somatic mutations in MYD88 (95-97\%) and CXCR4 (30-40\%) in WM patients. ${ }^{2,3}$ Mutated MYD 88 triggers NF- $\mathrm{KB}$ pro-survival signaling via Bruton's tyrosine kinase (BTK) and interleukin-1 receptor-associated kinase 1 (IRAK1)/IRAK4, and transactivates hematopoietic cell kinase (HCK). ${ }^{4,5}$ Both BTK and HCK are targeted by ibrutinib. ${ }^{4,5}$ Mutations in the C-terminal domain of CXCR4 are typically subclonal and support intrinsic ibrutinib resistance through upregulation of AKT and ERK1/2 signaling. ${ }^{6-9}$

In 2015, ibrutinib became the first approved agent by the United States Food and Drug Administration and European Medicines Agency for the treatment of symptomatic WM patients. The regulatory approval of ibrutinib was based on the results from a multi-center, single-arm, phase II trial of 63 previously treated WM patients. ${ }^{10}$ Ibrutinib monotherapy was highly active with an overall response rate
Haematologica 2022

Volume 107(5):1163-1171

\section{Correspondence:}

JORGE J. CASTILLO

jorgej_castillo@dfci.harvard.edu

Received: April 28, 2021.

Accepted: June 16, 2021.

Pre-published: June 24, 2021.

https://doi.org/10.3324/haematol.2021.279112

(C)2022 Ferrata Storti Foundation

Material published in Haematologica is covered by copyright. All rights are reserved to the Ferrata Storti Foundation. Use of published material is allowed under the following terms and conditions:

https://creativecommons.org/licenses/by-nc/4.0/legalcode. Copies of published material are allowed for personal or internal use. Sharing published material for non-commercial purposes is subject to the following conditions: https://creativecommons.org/licenses/by-nc/4.0/legalcode, sect. 3. Reproducing and sharing published material for commercial purposes is not allowed without permission in writing from the publisher. 
(ORR) of $91 \%$, major response rate (MRR) of $79 \%$, and very good partial response rate (VGPR) of $30 \%$ with prolonged follow-up. ${ }^{10,11}$ Responses to ibrutinib were durable with an estimated 5-year progression-free survival (PFS) and overall survival (OS) of $54 \%$ and $87 \%$, respectively. A notable finding was the impact of MYD 88 and CXCR4 mutations on ibrutinib outcomes. Patients wild-type (WT) for both MYD88 and CXCR4 had no major responses and a median PFS of 5 months to ibrutinib. ${ }^{10-12}$ Among patients with mutated MYD88, the concurrent presence of a CXCR4 mutation adversely impacted response rates, response kinetics, and 5-year PFS (38\% vs. $70 \%)^{10,11}$ Similar outcomes to ibrutinib monotherapy have been reported in phase II trials of treatment-naive $(n=30)$ and rituximab-refractory WM patients $(\mathrm{n}=31)$, as well as in the recent phase III ASPEN trial $(\mathrm{n}=199)$ comparing ibrutinib to zanubrutinib. ${ }^{13-16}$

Despite the high response rates and durable remissions, acquired ibrutinib resistance is increasingly being observed in WM patients. Approximately half of WM patients who progress on ibrutinib acquire BTK mutations at the binding site of ibrutinib (BTK C481S) or its downstream mediator PLC $\gamma 2 .{ }^{17}$ BTK C481S mutations are highly subclonal and confer protection to BTK WT clones via a paracrine mechanism. ${ }^{17,18}$ Acquired deletions in $6 \mathrm{q}$ and $8 p$ that contain regulators of BTK, MYD88/NF-KB, and apoptotic signaling also occur. ${ }^{19}$ However, data on the clinical outcomes of WM patients who progress while on active ibrutinib therapy are limited. Preliminary studies have described an abrupt increase in serum IgM level (i.e., IgM rebound) in some WM patients who discontinue ibrutinib. ${ }^{20,21}$ We sought to further characterize the clinical presentation, management, and outcomes of WM patients with acquired ibrutinib resistance, as well as the impact of BTK C481S mutations.

\section{Methods}

\section{Study design and patient selection}

We reviewed a prospectively maintained database of 362 patients seen at our institution between January 2012 and October 2020 who met clinicopathological criteria for WM and received ibrutinib monotherapy. ${ }^{1}$ Patients who had disease progression on active ibrutinib therapy per consensus guidelines were identified and included in this study. ${ }^{22} \mathrm{~A}$ transient increase in serum IgM level associated with a temporary hold of ibrutinib was not considered disease progression. The date a patient discontinued ibrutinib because of disease progression was defined as time-zero $\left(T_{0}\right)$. Pertinent clinical and pathological data were gathered for all patients at the time of $T_{0}$ until the last follow-up or death. The Dana-Farber/Harvard Cancer Center Institutional Review Board approved this study, and all patients provided written consent.

\section{Response and outcome definitions}

We defined an IgM rebound as a $\geq 25 \%$ increase in serum IgM level following $\mathrm{T}_{0}$, with an absolute increase of at least $500 \mathrm{mg} / \mathrm{dL}$, consistent with previous studies. ${ }^{20,21}$ Response assessment to salvage therapy was performed according to consensus guidelines from the $6^{\text {th }}$ International Workshop on WM. ${ }^{22}$ The ORR was defined as a minor response or better $(225 \%$ reduction in serum IgM level), and the MRR was defined as a partial response or better $(250 \%$ reduction in serum IgM level). Consensus guidelines were also utilized to assess response to salvage therapy for patients with light chain (AL) amyloidosis and diffuse large B cell lymphoma (DLBCL). ${ }^{23,24}$ The ORR and MRR were assessed for each regimen used after $T_{0}$. Duration of response (DOR) was defined as the length of time between response attainment and progression, death, or last follow-up. Survival after disease progression on ibrutinib was defined as the length of time between $T_{0}$ and the date of death or last follow-up.

\section{Tumor genotyping}

The presence of MYD88, CXCR4, and BTK mutations was detected by allele-specific polymerase chain reaction (AS-PCR) and Sanger sequencing methods, as previously described. ${ }^{6,17,25} \mathrm{~A}$ clinically validated next-generation sequencing (NGS) assay was also performed in a subset of patients on unselected bone marrow (BM) aspirate samples to identify TP53 mutations. ${ }^{26}$

\section{Statistical analyses}

Patient characteristics were summarized using descriptive statistics. Continuous variables were dichotomized using standard WM cutoffs to facilitate analysis, and comparisons were made using the $\chi^{2}$ test or Fischer exact test depending on the number of observations. Univariate and multivariate logistic regression models were utilized to identify predictive factors for an IgM rebound and response to salvage therapy; the outcome measure was odds ratio (OR) with $95 \%$ confidence interval (CI). Time to events was estimated using the Kaplan-Meier method, and comparisons between groups were made using the log-rank test. The Cox-proportional hazard regression method was used to fit univariate and multivariate models for OS; the outcome measure was hazard ratio (HR) with $95 \% \mathrm{CI}$. $P$-values were two-sided and considered statistically significant if $<0.05$. All calculations and graphs were obtained using R (R Foundation for Statistical Computing, Vienna, Austria).

\section{Results}

\section{Patient characteristics}

We identified $51 \mathrm{WM}$ patients with acquired resistance to ibrutinib monotherapy whose findings are included in this study. The baseline clinical characteristics at $T_{0}$ are summarized in Table 1. The median duration between WM diagnosis and study entry $\left(\mathrm{T}_{0}\right)$ was 8.2 years (range, $0.5-24$ years). The median treatment duration with ibrutinib before $\mathrm{T}_{0}$ was 2.0 years (range, 0.4-6.5 years). The median time between disease progression on ibrutinib and $\mathrm{T}_{0}$ was 25 days (range, $0-426$ days); seven patients (14\%) deriving clinical benefit continued on ibrutinib for $>90$ days after meeting criteria for disease progression before discontinuing therapy. Forty-three patients (84\%) had received ibrutinib in the relapsed or refractory setting, and eight $(16 \%)$ in the frontline setting. The median number of treatment lines including ibrutinib before $T_{0}$ was four (range, 1-9). Twenty patients (39\%) were previously exposed to the major drug classes during their disease course, including rituximab, proteasome inhibitors, alkylators, and ibrutinib (i.e., "quadruple-class exposed"). MYD 88 and CXCR4 mutations were present in 93\% and $58 \%$ of genotyped patients, respectively, and the majority (87\%) of CXCR4 mutations were nonsense variants. The clinical manifestations at the time of disease progression on ibrutinib showed considerable heterogeneity and are presented in Table 2.

\section{Serum immunoglobulin $\mathbf{M}$ rebound}

The peak absolute change in serum IgM level following 
$\mathrm{T}_{0}$ for each patient is shown in Figure 1. An IgM rebound occurred in 29 of $48(60 \%)$ evaluable patients following $\mathrm{T}_{0}$. Three patients who developed symptomatic hyperviscosity while progressing on ibrutinib received plasmapheresis immediately before and after $T_{0}$ and were deemed non-evaluable for an IgM rebound. The median time to an IgM rebound was 27 days (95\% CI: 24-33;

Table 1. Baseline characteristics at time of ibrutinib discontinuation $\left(\mathrm{T}_{0}\right)$.

$\begin{array}{lc}\text { Patient characteristic } & \text { All patients }(n=51) \\ \text { Median age (range) }- \text { yrs } & \\ \text { WM diagnosis } & 59(40-91) \\ \text { Ibrutinib initiation } & 66(43-93) \\ \text { Ibrutinib discontinuation } & 69(43-93) \\ \text { Time from WM diagnosis } & \\ \text { Median (range) - yrs } & 8.2(0.5-24) \\ >10 \text { yrs - no. (\%) } & 20(39)\end{array}$

Time from ibrutinib initiation

$\begin{array}{lc}\text { Median (range) - yrs } & 2(0.4-6.5) \\ >2 \text { yrs - no. (\%) } & 25(49)\end{array}$

Time from disease progression on ibrutinib

\begin{tabular}{lc} 
Median (range) - days & $25(0-426)$ \\
$>90$ days - no. (\%) & $7(14)$ \\
\hline Male sex - no. (\%) & $33(65)$ \\
Hemoglobin level & \\
Median (range) - g/dL & $10.3(7.3-16.6)$ \\
$<10 \mathrm{mg} / \mathrm{dL}-$ no. $(\%)$ & $20(39)$
\end{tabular}

Platelet count

$\begin{array}{lc}\text { Median (range) }-\mathrm{K} / \mathrm{uL} & 171(7-463) \\ <100 \mathrm{~K} / \mathrm{uL}-\text { no. }(\%) & 16(31)\end{array}$

Serum IgM level

$\begin{array}{cc}\text { Median (range) }-\mathrm{mg} / \mathrm{dL} & 1,567(97-7,935) \\ >4000 \mathrm{mg} / \mathrm{dL}-\text { no. }(\%) & 9(18)\end{array}$

Bone marrow involvement

$\begin{array}{lc}\text { Median (range) - \% } & 70(5-90) \\ >50 \%-\text { no. }(\%) & 16(70)\end{array}$

Previous therapy (including ibrutinib)

Median no. of treatment lines (range)

Treatment lines - no. (\%)

$1-2$

$3-4$

$5+$

Types of therapy - no. (\%)

IB

$\mathrm{IB}+\mathrm{R}$

$\mathrm{IB}+\mathrm{R}+\mathrm{PI}$

$10(20)$

$\mathrm{IB}+\mathrm{R}+$ alkylator

$\mathrm{IB}+\mathrm{R}+\mathrm{PI}+$ alkylator

MYD88 mutation - no. (\%)

CXCR4 mutation - no. (\%)

$20(39)$

43 (93)

23 (58)

Nonsense

$20(87 \%)$

Frameshift

Data on bone marrow involvement at the time of ibrutinib relapse was available for 23 patients. MYD88 and CXCR4 mutation status was available for 46 and 40 patients, respectively. IB: ibrutinib; R: rituximab; PI: proteasome inhibitor. WM: Waldenström macroglobulinemia.
Figure $2 \mathrm{~A})$. The cumulative incidence of an IgM rebound following $\mathrm{T}_{0}$ increased over time: 7 days (9\%); 14 days (13\%); 21 days (25\%); 28 days (46\%); and 35 days (65\%). Patients with an IgM rebound had a peak median absolute and relative increase in serum IgM level of $1,405 \mathrm{mg} / \mathrm{dL}$ (range, 571-7,820 $\mathrm{mg} / \mathrm{dL}$ ) and 79\% (range, $27-1,663 \%$ ), respectively. The degree of $B M$ involvement at $T_{0}$ significantly correlated with both the absolute $(\mathrm{r}=0.44 ; P=0.047)$ and relative $(r=0.45 ; P=0.04)$ changes in serum IgM level. Twenty-one patients ( $72 \%$ ) had an increase in serum IgM level back to the pre-ibrutinib baseline or higher. Symptomatic hyperviscosity acutely developed after $\mathrm{T}_{0}$ in ten of 29 patients (34\%) with an IgM rebound that prompted emergent plasmapheresis. The median time from $\mathrm{T}_{0}$ to the onset of symptomatic hyperviscosity was 29 days (range, 14-51 days). Serial IgM measurements were available for seven of ten $(70 \%)$ patients that developed symptomatic hyperviscosity and are shown in Online Supplementary Figure S1. Seven patients (24\%) had an IgM rebound present during the first cycle of salvage therapy; none of these patients were receiving rituximab concurrently.

The timing of salvage therapy following $T_{0}$ impacted the risk of an IgM rebound. Patients who received salvage therapy $\leq 7$ versus $>7$ days from $\mathrm{T}_{0}$ had significantly lower odds of an IgM rebound ( $29 \%$ vs. $76 \%$; OR $0.15,95 \% \mathrm{CI}$ : $0.03-0.67 ; P=0.005)$. Bridging ibrutinib with salvage therapy was also associated with significantly lower odds of an IgM rebound compared to no bridging (17\% vs. 69\%; OR $0.10,95 \%$ CI: $0.01-0.97 ; P=0.03)$. There was a trend for lower odds of an $\operatorname{IgM}$ rebound when bridging ibrutinib versus starting salvage therapy within 7 days of $\mathrm{T}_{0}(17 \%$ vs. $43 \%$; OR 0.11, 95\% CI: 0.01-1.19; $P=0.11)$. We were unable to identify any factor at $\mathrm{T}_{0}$ predictive of an $\mathrm{IgM}$ rebound. Age, time on ibrutinib, time from WM diagnosis, sex, hemoglobin level, platelet count, serum IgM level, number and type of previous therapies, and MYD 88 and CXCR4 mutation status were not associated with higher or lower odds of an IgM rebound $(P>0.05$ for all comparisons; Online Supplementary Table S1).

\section{Salvage therapy}

Forty-eight patients (94\%) received salvage therapy following $T_{0}$. The median time to salvage therapy was 18

Table 2. Clinical manifestations of disease progression on ibrutinib.

\begin{tabular}{lc}
\hline Feature & All patients ( $\mathbf{l}=51$ ) \\
Progressive cytopenias & $22(43)$ \\
Lymphadenopathy and/or splenomegaly & $12(24)$ \\
\hline Malignant pleural effusion & $6(12)$ \\
Cardiac AL amyloidosis & $5(10)$ \\
\hline Isolated serum IgM increase & $4(8)$ \\
Symptomatic hyperviscosity & $4(8)$ \\
\hline Soft tissue mass & $4(8)$ \\
Bing-Neel syndrome & $3(6)$ \\
\hline DLBCL transformation & $2(4)$ \\
Malignant pericardial effusion & $1(2)$ \\
\hline Renal monoclonal IgM deposition & $1(2)$ \\
\hline
\end{tabular}

Patients may have had more than one manifestation of disease progression on ibrutinib. Soft tissue masses developed in the palate, orbit, maxilla, and thoracic spine with cord displacement ( $\mathrm{n}=1$ for each). None of the patients with histological transformation were previously treated with a nucleoside analogue. AL: light chain amyloidosis; IgM: immunoglobulin M; DLBCL: diffuse large B-cell lymphoma. 
days (95\% CI: 13-27); treatment was started within 4 and 8 weeks of $\mathrm{T}_{0}$ for $69 \%$ and $93 \%$ of patients, respectively (Figure 2B). Reasons for not receiving salvage therapy included patient choice of hospice care $(n=2)$ and decompensated heart failure from cardiac AL amyloidosis $(n=1)$. The ORR and MRR to the first salvage regimen following $\mathrm{T}_{0}$ were $56 \%(27 / 48)$ and $44 \%$ (21/48), respectively. Among patients who responded to salvage therapy, the median DOR was 48 months (34 months-NR), and the 3 -year DOR was $61 \%$ (41-90\%). Twenty patients were refractory $(42 \%)$ to the first salvage regimen; 11 patients received subsequent treatment, and nine patients died from progressive disease before receiving additional treatment. The specific treatment regimens utilized for the first salvage regimen after $T_{0}$ with the corresponding response rates and DOR are detailed in Table 3.

We then performed additional analyses to identify factors at $T_{0}$ predictive of a response to the first salvage regimen. Patients with quadruple-class (rituximab, proteasome inhibitor, alkylator, ibrutinib) exposed disease had significantly lower odds of a response to the first salvage regimen compared to those without (33\% vs. $73 \%$; OR 0.18 , 95\% CI: 0.04-0.76; $P=0.01$ ). Age, time on ibrutinib, time from WM diagnosis, sex, hemoglobin level, platelet count, serum IgM level, number of previous therapies, and MYD 88 and CXCR4 mutation status were not associated with higher or lower odds of a response to the first salvage regimen following $\mathrm{T}_{0}(P>0.05$ for all comparisons; Online Supplementary Table S2).

The timing of salvage therapy following $T_{0}$ also impacted the likelihood of a response to the first salvage regimen. Patients who received salvage therapy $\leq 7$ versus $>7$ days from $\mathrm{T}_{0}$ had significantly higher odds of a response $(75 \%$ vs. $45 \%$; OR 4.47, 95\% CI: 1.07-23.2; $P=0.03$ ). Bridging ibrutinib with salvage therapy was also associated with a significantly higher response rate $(100 \%$ vs. $49 \%$; $P=0.01)$. There was a trend for a higher response rate with ibrutinib bridging versus initiating salvage therapy within 7 days of $\mathrm{T}_{0}(100 \%$ vs. $58 \% ; P=0.054)$.

In a multivariate model, we evaluated quadruple-class exposed disease against receiving salvage therapy $\leq 7$ days after $T_{0}$ for the odds of a response to salvage therapy. Both quadruple-class exposed disease (OR 0.20, 95\% CI: $0.05-$ $0.73 ; P=0.02)$ and receiving salvage therapy $\leq 7$ days after $\mathrm{T}_{0}$ (OR 4.12, 95\% CI: 1.07-18.9; $P=0.048$ ) remained inde- pendently associated with the odds of attaining a response to salvage therapy.

Eight patients bridged ibrutinib with the subsequent treatment. Ibrutinib overlapped with the salvage regimen for two cycles in six patients, and one cycle in two patients. The following treatment regimens were added while continuing ibrutinib: bendamustine and rituximab (Benda-R; $n=3$ ), bortezomib, dexamethasone, and rituximab (BDR; n=3), ixazomib, dexamethasone, and rituximab (IDR; $n=1$ ), and fludarabine and rituximab (Flu-R; $\mathrm{n}=1)$. The ORR and MRR to bridging ibrutinib with salvage therapy were both $100 \%$. Six patients were evaluable for an IgM rebound; two patients had developed symptomatic hyperviscosity as part of clinical progression on ibrutinib and were deemed unevaluable for an IgM rebound. Only one patient $(17 \%)$ had an asymptomatic IgM rebound after bridging ibrutinib with Benda- $\mathrm{R}$ for two cycles, which subsequently resolved with two additional treatment cycles. The two non-evaluable patients with symptomatic hyperviscosity were able to stop plasmapheresis after one cycle of bridging with BDR, and then discontinued ibrutinib without evidence of an IgM rebound following one additional cycle of bridging. Ibrutinib was bridged with Flu-R in one patient with BingNeel syndrome, and there was no evidence of an IgM rebound or worsening of neurological symptoms follow-

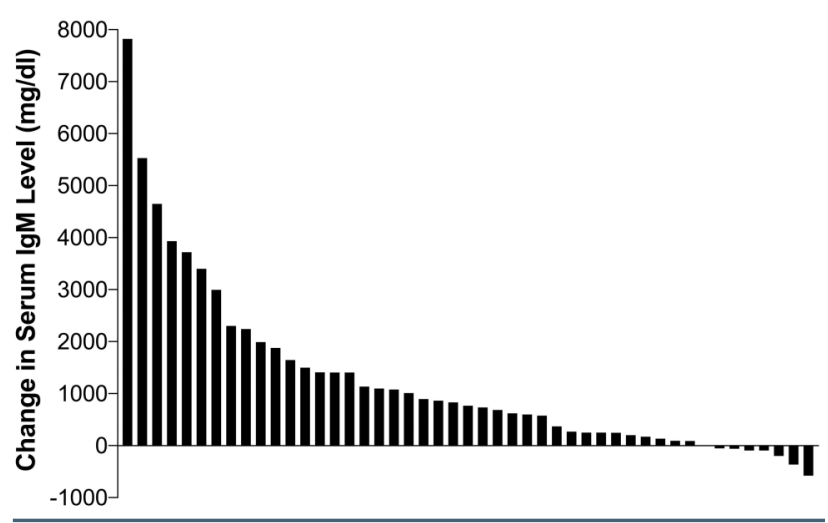

Figure 1. Peak absolute change in serum immunoglobulin M level following discontinuation of ibrutinib. Values are depicted for each of the 48 patients who were evaluable for an immunoglobulin M (IgM) rebound.

Table 3. Response outcomes according to each salvage regimen utilized following ibrutinib discontinuation.

\begin{tabular}{|c|c|c|c|c|}
\hline Salvage Regimen & $\mathbf{N}$ & $\begin{array}{l}\geq \text { Minor Response } \\
\text { (ORR) }\end{array}$ & $\begin{array}{l}\geq \text { Partial Response } \\
\text { (MRR) }\end{array}$ & DOR (months) \\
\hline Benda-R & 22 & $14(64)$ & $11(50)$ & 3-yr: $57 \%$ \\
\hline PI-Dex-R & 8 & $5(63)$ & $4(50)$ & 3-yr: 80\% \\
\hline Flu-R & 4 & $4(100)$ & $3(75)$ & 3-yr: $67 \%$ \\
\hline CCD & 2 & $1(50)$ & $1(50)$ & $2.7+$ \\
\hline R-CHOP & 2 & $1(50)$ & $1(50)$ & $26+$ \\
\hline Ofatumumab & 1 & $1(100)$ & $0(0)$ & 15 \\
\hline Daratumumab & 2 & $0(0)$ & $0(0)$ & - \\
\hline Ibrutinib + PI & 1 & $0(0)$ & $0(0)$ & - \\
\hline Everolimus & 1 & $0(0)$ & $0(0)$ & - \\
\hline
\end{tabular}

A total of 48 of 51 patients (94\%) received at least one salvage therapy following time-zero $\left(\mathrm{T}_{0}\right)$. Five patients received investigational agents and the responses are not included in the table. One patient with AL amyloidosis received consolidation with an autologous stem cell transplant following Benda-R.The following proteasome inhibitors were used as part of a PI-Dex-R regimen: bortezomib ( $n=5)$; carfilzomib $(n=2)$; ixazomib $(n=1)$. ORR: overall response rate; MRR: major response rate; DOR: duration of response; yr: years PI-Dex-R: proteasome inhibitor, dexamethasone, rituximab; Flu-R: fludarabine, rituximab; CCD: carfilzomib, cyclophosphamide, dexamethasone; R-CHOP: rituximab, cyclophosphamide, doxorubicin, vincristine, prednisone. 
A

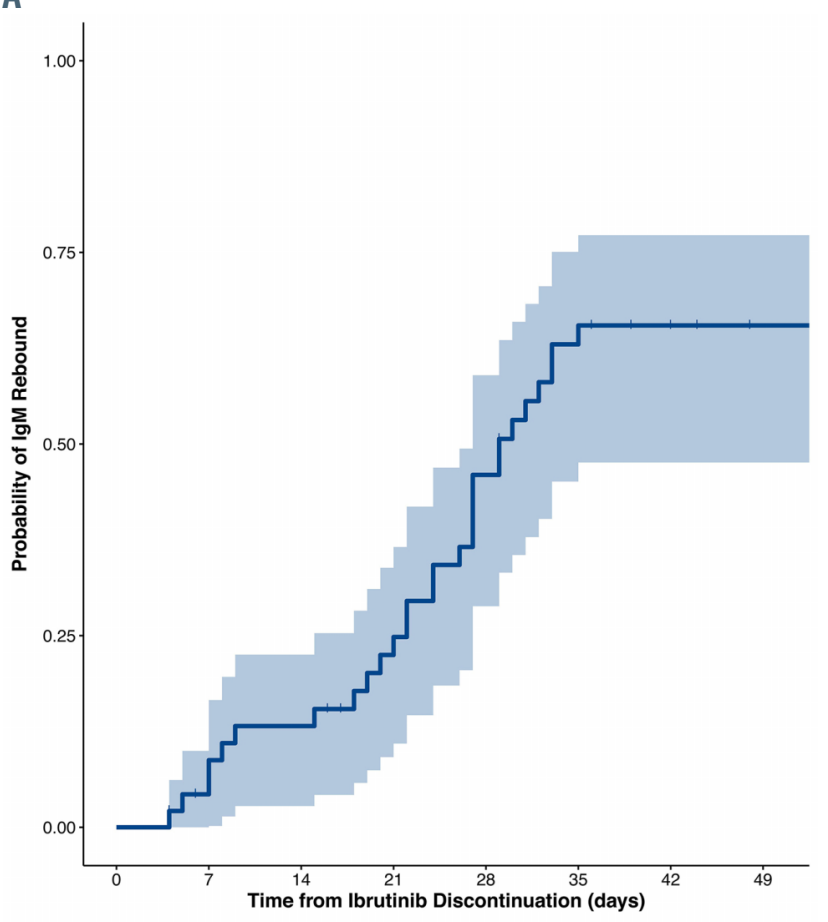

Number at risk

B

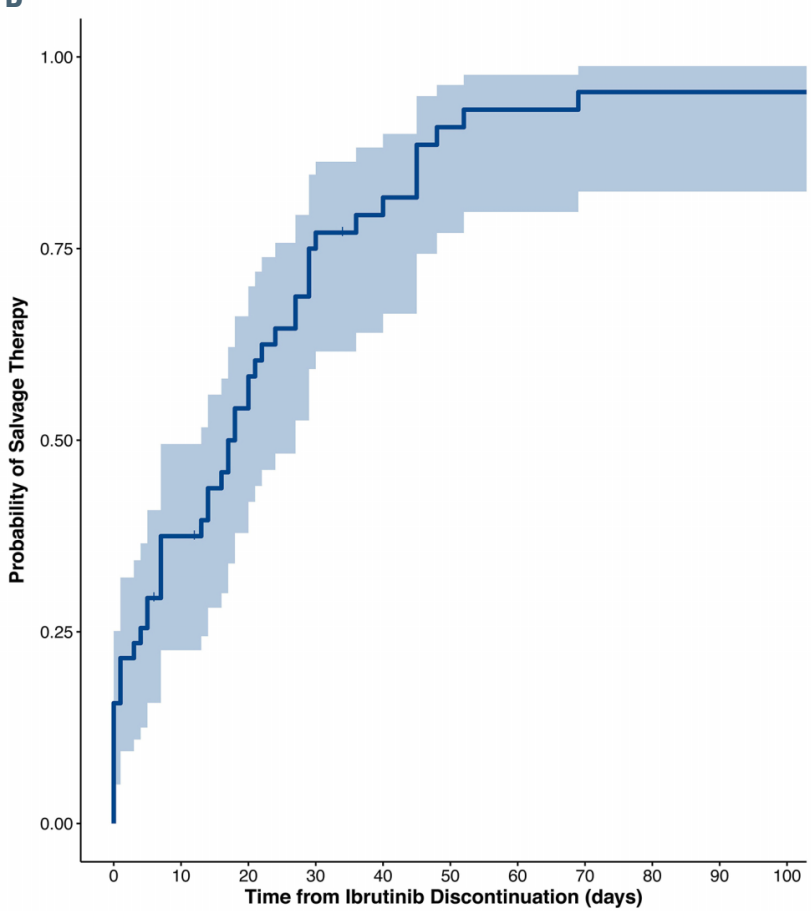

Number at risk

51

Figure 2. Estimated cumulative incidence of an immunoglobulin $M$ rebound $(A)$ and salvage therapy (B) following discontinuation of ibrutinib. An immunoglobulin M (IgM) rebound occurred in 29 of $48(60 \%)$ evaluable patients. The median time to an IgM rebound was 27 days (95\% confidence interval [CI]: 24-33 days). Forty-eight patients $(94 \%)$ received salvage therapy following time-zero $\left(T_{0}\right)$. The median time to salvage therapy was 18 days (95\% Cl: $13-27$ days). ing $T_{0}$. Bridging ibrutinib with salvage therapy was well tolerated, and no unexpected toxicities were observed.

\section{Survival outcomes}

The median follow-up from $\mathrm{T}_{0}$ was 13 months (range, 0.2-75 months) for the entire cohort, and 20 patients (39\%) had died at the time of this report. The median OS from $T_{0}$ was 51 months (95\% CI: 15.3-not reached [NR]), and the 5year OS was 44\% (95\% CI: 26-75) (Figure 3A). The median OS for the patients who received at least one salvage regimen after $\mathrm{T}_{0}$ was 51 months (95\% CI: 21-NR). Patients who did not receive any salvage therapy following $T_{0}(n=3)$ had a median OS of 0.4 months (95\% CI: $0.20-N R)$, with survival times of $0.2,0.8$, and 0.4 months, respectively. The median OS from WM diagnosis for the entire cohort was 20.4 years (95\% CI: 13.2-NR; Online Supplementary Figure S2).

The prognostic factors identified in a univariate analysis that impact OS after $T_{0}$ are shown in Table 4. Only the types of previous therapy received before $T_{0}$ significantly impacted $\mathrm{OS}(P=0.018$; Figure $3 \mathrm{~B})$. Quadruple-class (rituximab, proteasome inhibitor, alkylator, ibrutinib) exposed disease was significantly associated with a shorter OS following $\mathrm{T}_{0}$ (HR 8.08, 95\% CI: 1.05-6.21; $P=0.04$ ). Among patients without quadruple-class exposed disease, there was no significant difference in OS between the different types of previous therapy $(P=0.57)$. Patients with and without quadruple-class exposed disease had a median OS following $\mathrm{T}_{0}$ of 13.2 months and $\mathrm{NR}$, respectively $(P<0.001$; Online Supplementary Figure S3). The 5-year OS for patients without quadruple-class exposed disease was $62 \%$ (95\% CI: 38-98).

OS was impacted by the attainment and depth of response to the first salvage regimen after $T_{0}$. The median OS was significantly longer among patients who achieved a response to the first salvage regimen versus those patients who did not (NR vs. 10.8 months; 95\% CI: 0.010.27 ; $P<0.0001$; Figure $3 \mathrm{C})$. When stratified by the depth

Table 4. Prognostic factors for overall survival at the time of ibrutinib discontinuation $\left(\mathrm{T}_{0}\right)$.

\begin{tabular}{lcc}
\hline Patient characteristic & HR $(95 \%$ CI) & $P$ \\
Age $>65$ yrs. & $1.26(0.50-3.18)$ & 0.62 \\
$>10$ yrs from WM diagnosis & $1.60(0.66-3.87)$ & 0.30 \\
\hline Male sex & $1.53(0.55-4.22)$ & 0.41 \\
Hemoglobin level $<10 \mathrm{mg} / \mathrm{dL}$ & $2.12(0.85-5.26)$ & 0.11 \\
\hline Platelet count $<100,000 / \mu \mathrm{L}$ & $1.92(0.76-4.81)$ & 0.17 \\
Serum IgM level $>4000 \mathrm{mg} / \mathrm{dL}$ & $0.51(0.12-2.20)$ & 0.37 \\
\hline$>4$ prior therapies & $2.08(0.75-5.78)$ & 0.16 \\
Type of previous therapy & & \\
IB & Reference & - \\
IB + R & $1.45(0.09-23.40)$ & 0.80 \\
IB + R + PI & $3.29(0.35-30.7)$ & 0.30 \\
IB + R + alkylator & $1.20(0.07-19.4)$ & 0.90 \\
IB + R + PI + alkylator & $8.08(1.05-62.1)$ & 0.04 \\
\hline MYD8 mutation & $0.55(0.12-2.34)$ & 0.41 \\
CXCR4 mutation & $1.36(0.57-3.27)$ & 0.49
\end{tabular}

The number of previous treatment lines includes ibrutinib monotherapy for al patients. The "types of previous therapy" variable summarizes the different classes of anti-neoplastic agents received throughout the Waldenström macroglobulinemia disease course for each patient. MYD88 and CXCR4 mutation status was available for 46 and 40 patients, respectively. IB: ibrutinib; R: rituximab; PI: proteasome inhibitor, yrs: years; HR: hazard ratio; $\mathrm{Cl}$ : confidence interval. 


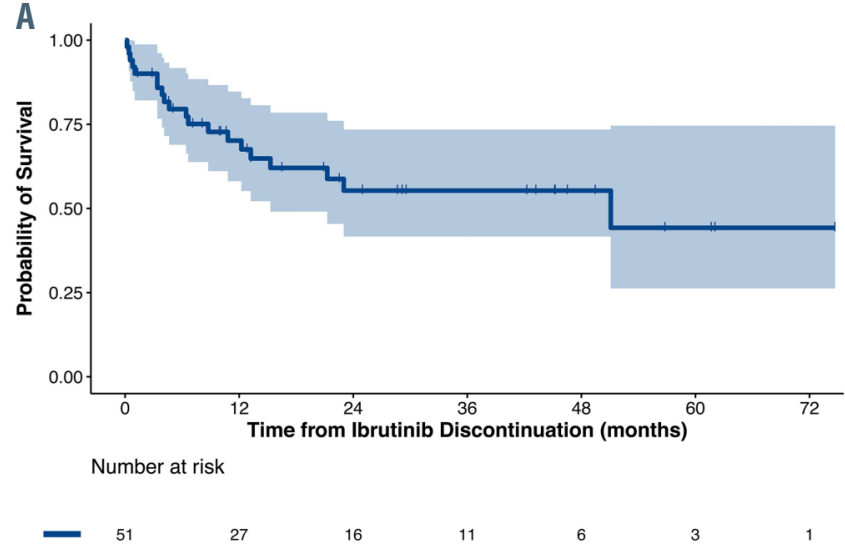

C

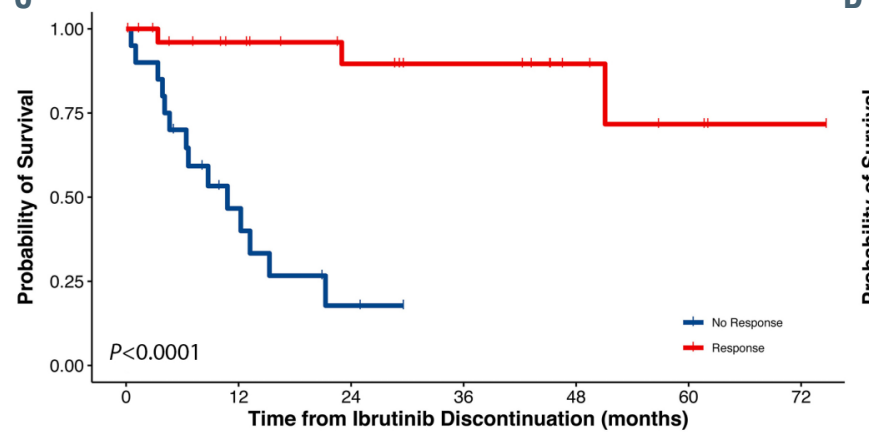

Number at risk

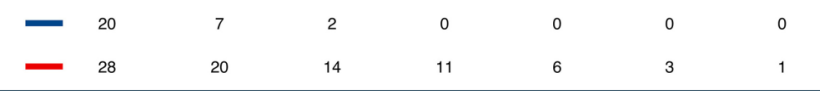

D
B
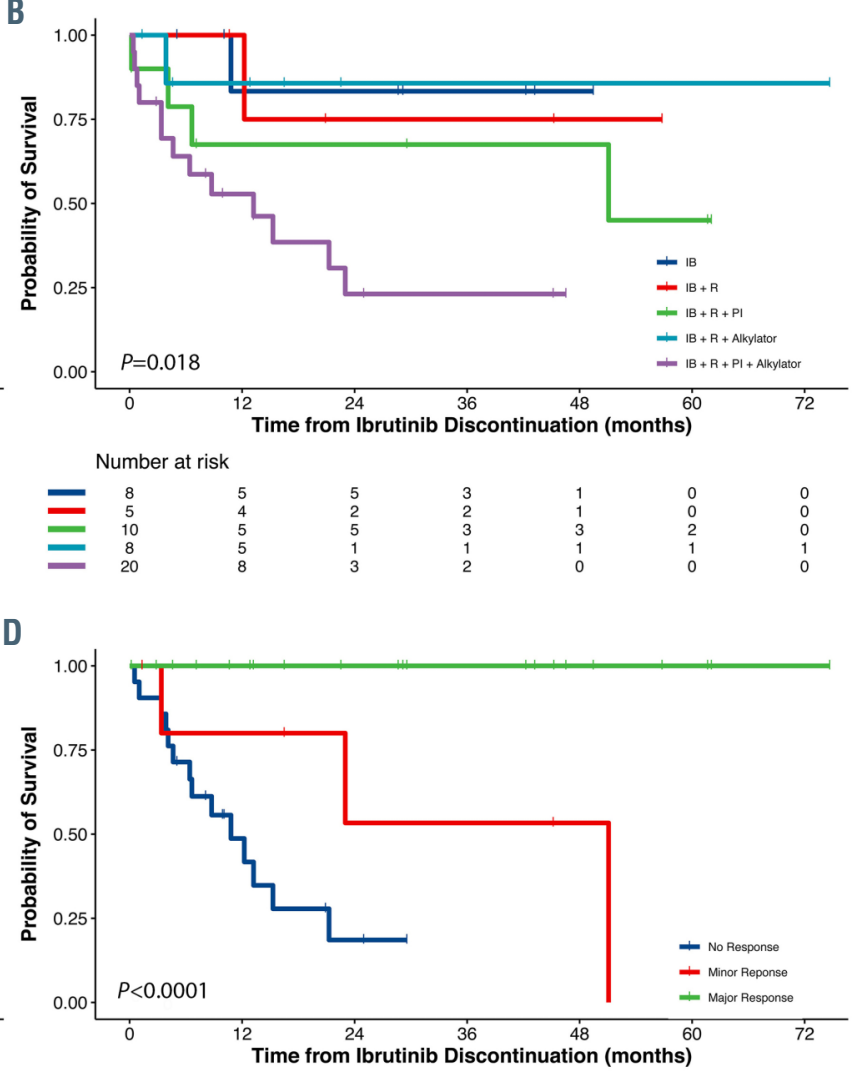
Number at risk

\begin{tabular}{|c|c|c|c|c|c|c|}
\hline 21 & 7 & 2 & 0 & 0 & 0 & 0 \\
\hline 6 & 4 & 2 & 2 & 1 & 0 & 0 \\
\hline 21 & 16 & 12 & 9 & 5 & 3 & 1 \\
\hline
\end{tabular}

Figure 3. Overall survival following ibrutinib discontinuation in resistant Waldenström macroglobulinemia patients. Kaplan-Meier overall survival curves following discontinuation of ibrutinib for the entire cohort (A) and stratified by types of previous therapy (B), response attainment to first salvage regimen (C), and depth of response to first salvage regimen (D). All patients were previously treated with ibrutinib monotherapy. The "types of previous therapy" variable summarizes the different classes of anti-neoplastic agents received throughout the Waldenström macroglobulinemia disease course for each patient. IB: ibrutinib; R: rituximab; PI: proteasome inhibitor.

of response, the median OS for patients who achieved a major response, minor response, and no response were NR (95\% CI: NR-NR), 51.1 months (95\% CI: 23-NR), and 10.8 months (95\% CI: 6.4-NR), respectively $(P<0.001$; Figure $3 \mathrm{D})$. The 5 -year OS for patients who achieved a major response to the first salvage regimen was $100 \%$. We then evaluated the presence of quadruple-class exposed disease against attaining a response to the first salvage regimen in a multivariate model for OS following $T_{0}$. Only a response to salvage therapy remained independently associated with OS (HR 0.08, 95\% CI: 0.02-0.38; $P=0.002$ ), whereas the presence of quadruple-class exposed disease had no impact $(P=0.20)$.

\section{Acquired BTK C481S mutations}

$B T K$ mutation testing was performed in 21 patients. Seven patients (33\%) had a BTK C481S mutation, including one patient with three different BTK C481S variants. There was no difference in the time to ibrutinib discontinuation $\left(\mathrm{T}_{0}\right)$ between patients with BTK C481S and BTK WT (1.9 vs. 1.8 years; $P=0.50$; Online Supplementary Figure S4). There was also no difference in age, time from WM diagnosis, sex, hemoglobin level, platelet count, serum IgM level, number or type of prior therapies, and MYD 88 and CXCR4 mutation status between patients with BTK
C481S and BTK WT ( $P>0.05$ for all comparisons; Online Supplementary Table S3). Likewise, BTK C481S was not associated with higher or lower odds of an IgM rebound $(P=0.99)$ or response to the first salvage regimen after $T_{0}$ $(P=0.16)$.

By univariate analysis, patients with BTK C481S had a significantly shorter median OS following $\mathrm{T}_{0}$ versus BTK WT (6.4 months vs. NR; $P=0.026$; Online Supplementary Figure 55 ). In an exploratory analysis, we evaluated the presence of BTK C481S against quadruple-class exposed disease for $O S$ after $T_{0}$. In this model, only quadruple-class exposed disease was significantly associated with worse OS (HR 5.50, 95\% CI: 1.15-26.2; P=0.03). BTK C481S was not independently associated with $O S$ after adjusting for quadruple-class exposed disease $(P=0.09)$.

\section{TP53 mutations}

Three of 20 patients $(15 \%)$ had a TP53 mutation detected. Two TP53 mutations were detected in one patient, and all TP53 mutations localized to the DNA-binding domain. All three patients had mutated MYD88, and two patients had a CXCR4 mutation; no concurrent BTK mutations were identified in the two patients tested. All three patients with a TP53 mutation had an IgM rebound following $\mathrm{T}_{0}$. No patient with a TP53 mutation responded to 
salvage therapy, and all were quadruple-class exposed. Patients with a TP53 mutation had a significantly shorter median OS following $T_{0}$ versus those without (0.5 vs. 21.3 months; $P=0.02$; Online Supplementary Figure S6).

\section{Discussion}

In this study, we sought to describe the natural history of WM patients who acquired resistance to ibrutinib monotherapy. Despite the high response rates and durable remissions, acquired ibrutinib resistance represents an emerging problem in WM patients, and understanding the subsequent disease course may help direct management strategies. Central to our findings was that stopping ibrutinib in resistant WM patients heralded rapid disease progression, which prompted the need for salvage therapy to achieve disease control. This contrasts the indolent posttreatment course typically observed in WM patients following rituximab-based regimens. ${ }^{27-30}$ Withholding ibrutinib temporarily for adverse events or procedures can also lead to acute increases in serum IgM level, anemia, and constitutional symptoms, highlighting the capacity of tumoral cells to rapidly disseminate disease following ibrutinib withdrawal. ${ }^{10,13,20,31,32}$

The exact mechanism driving the rapid disease progression after ibrutinib cessation remains to be clarified. However, the BTK substrate STAT5A regulates IgM secretion in WM cells, and its selective reactivation following ibrutinib withdrawal likely contributes to the rapid increase in serum IgM level observed. ${ }^{33,34}$ In addition, acquired ibrutinib resistance is associated with the clonal expansion of $B T K$ and PLC $\gamma 2$ mutations that trigger prosurvival ERK1/2 signaling and cytokine release, as well as deletions in $6 q$ and $8 p$ that contain regulators of BTK, MYD88/NF-KB, and apoptotic signaling. ${ }^{.7-19}$ It is possible these molecular mechanisms mediating ibrutinib failure contribute to disease acceleration following ibrutinib withdrawal. Indeed, we previously observed a higher risk of rapid disease progression in WM patients discontinuing ibrutinib for acquired resistance versus intolerance, signifying differences in underlying disease biology. ${ }^{20} \mathrm{~A}$ similar observation has also been described in patients with chronic lymphocytic leukemia (CLL), wherein rapid increases in serum lymphocyte counts were reported after stopping ibrutinib (i.e., "CLL flare"). ${ }^{35,36}$ Additional investigation is needed to elucidate whether the rapid disease progression in WM patients is driven by a hypersecretory state, rapid tumor proliferation, or a combination of both. Evaluating both the BM tumor burden and transcriptional signature in WM cells before and after ibrutinib discontinuation would provide further mechanistic insights into this phenomenon.

Akin to previous studies, we observed the occurrence of an IgM rebound following discontinuation of ibrutinib. ${ }^{20,21}$ Rapid increases in serum IgM level can exacerbate WMrelated morbidity caused by the IgM paraprotein, including hyperviscosity, peripheral neuropathy, cold agglutinemia, and cryoglobulinemia. ${ }^{37}$ In this study, approximately one in three patients with an IgM rebound acutely developed symptomatic hyperviscosity and required emergent plasmapheresis. These findings indicate that close monitoring of serum IgM levels is necessary in WM patients immediately after stopping ibrutinib. Hyperviscosity prophylaxis with plasmapheresis may also warrant consider- ation in WM patients stopping ibrutinib with high serum IgM levels, as the risk of symptomatic hyperviscosity increases exponentially when the serum IgM level rises above $3,000 \mathrm{mg} / \mathrm{dL} \cdot{ }^{38} \mathrm{~A}$ similar approach is recommended in WM patients receiving rituximab-based therapy to mitigate the risk of hyperviscosity-related injury caused by an IgM flare. ${ }^{39,40}$

Our data suggest that early initiation of salvage therapy can forestall disease acceleration after stopping ibrutinib. This observation is clinically relevant given the impact of response attainment to salvage therapy on post-ibrutinib survival. Patients who received treatment within 1 week of ibrutinib discontinuation had a significantly lower risk of an IgM rebound, as well as higher response rates to salvage therapy. Notably, bridging ibrutinib in combination with the subsequent therapy for 1-2 cycles achieved an objective response in all patients, and may represent a strategy to maintain disease control in select patients. Similar efficacy with bridging has been reported in ibrutinib-resistant CLL patients who bridged ibrutinib with venetoclax. ${ }^{41}$ Taken together, these data support the recent consensus guidelines that recommend continuing ibrutinib until the subsequent therapy, plus consideration of bridging, in ibrutinib-resistant WM patients. ${ }^{42}$ Clinical trials should also consider allowing shorter wash-out periods or overlap of ibrutinib for WM patients in this clinical scenario.

The optimal treatment regimen for WM patients after ibrutinib has yet to be established in prospective studies. Our findings demonstrate that standard WM regimens such as Benda-R and BDR are effective as salvage therapy, especially in patients naive to these agents. Patients with quadruple-class exposed disease, by contrast, had inferior post-ibrutinib outcomes, likely reflecting the presence of a WM clone with little residual sensitivity to available therapies. Importantly, the BCL2 inhibitor venetoclax may represent a novel treatment option for WM patients. Preliminary results from a phase II trial evaluating venetoclax in relapsed or refractory WM patients reported an ORR of $87 \%$, MRR of $81 \%$, and 2 -year PFS of $76 \%$. Responses to venetoclax were attained in WM patients previously treated with ibrutinib, akin to studies evaluating venetoclax in ibrutinib-resistant CLL patients. ${ }^{43,44}$ Combination therapy with IDR or idelalisib plus obinutuzumab are alternative novel salvage regimens, but their activity following ibrutinib is currently unknown. ${ }^{45-48}$ Non-covalent BTK inhibitors, such as LOXO-305 (clinaltrials gov. Identifier: NCT03740529), vecabrutinib (clinaltrials gov. Identifier: NCT03037645), and ARQ-513 (clinaltrials gov. Identifier: NCT03162536), that bind to nonBTK C481S targets are also under investigation in WM patients. Lastly, a clinical trial is underway with the HCK inhibitor dasatinib for WM patients who are progressing on ibrutinib (clinaltrials gov. Identifier: NCT04115059).

Clinical trials have shown CXCR4 mutations confer resistance to ibrutinib monotherapy in WM patients, characterized by lower response rates, delayed response attainment, and shorter PFS. ${ }^{10-15,49}$ Consistent with these findings, our cohort of ibrutinib-resistant WM patients was enriched for CXCR4 mutations relative to the established incidence $\left(58 \%\right.$ vs. 30-40\%), ${ }^{3,6,50}$ Moreover, the majority of CXCR4 mutations were nonsense variants, supporting recent reports that this subtype of CXCR4 mutation shows greater resistance to ibrutinib monotherapy. ${ }^{11,51,52}$ Combination therapy with ibrutinib plus ritux- 
imab is also adversely impacted by CXCR4 mutations, with a shorter 36-month PFS in CXCR4 mutated versus CXCR4 WT WM patients (64\% vs. 84\%, respectively). ${ }^{53-55}$ Given the importance of CXCR4 mutations, clinical trials evaluating the CXCR4 inhibitors ulocuplumab (clinaltrials gov. Identifier: NCT03225716) and mavorixafor (clinaltrials gov. Identifier: NCT04274738) in combination with ibrutinib are currently ongoing in CXCR4-mutated WM patients.

A notable finding was the similar disease course between BTK C481S and BTK WT ibrutinib-resistant WM patients. It is possible a shared ERK1/2 signature underlies this clinical observation. In WM patients with BTK WT, $P L C \gamma 2$ mutations and DOK2 deletions were identified as possible molecular mechanisms driving acquired ibrutinib resistance. ${ }^{17,19}$ Both are predicted to trigger ERK1/2 signaling similar to the effect of BTK C481S mutations, ${ }^{18,56}$ although studies are needed to confirm the functional significance of $P L C \gamma 2$ and DOK2 in WM. These studies may also inform the utility of ERK1/2 inhibitors as a strategy to overcome acquired ibrutinib resistance in WM patients with BTK WT. The use of an ERK1/2 inhibitor has previously been shown to abrogate the effects of BTK C481S in WM cells and restore sensitivity to ibrutinib. ${ }^{18} \mathrm{We}$ also observed TP53 mutations were associated with refractory disease and shorter survival after acquiring resistance to ibrutinib. Although both preclinical and clinical data suggest ibrutinib has activity in TP53-mutated WM patients, additional work is needed to identify novel treatments for this high-risk group. ${ }^{57,58} \mathrm{~A}$ phase II trial evaluating ibrutinib in previously untreated WM patients with serial wholeexome sequencing is now complete and will provide additional insights into mechanisms of ibrutinib resistance, as well as the impact of ibrutinib on clonal evolution (clinicaltrials gov. Identifier: NCT02604511).

Limitations of this study include the inherent selection bias associated with a retrospective study from a single tertiary referral center. Nevertheless, this study constitutes the largest clinical experience of WM patients with acquired ibrutinib resistance, and the patients included are representative of those who participate in clinical trials This study can therefore serve as a "real-world" benchmark for assessing new drugs in WM patients with acquired ibrutinib resistance.

In conclusion, our findings show that discontinuation of ibrutinib can herald rapid disease progression in WM patients with acquired ibrutinib resistance. A rapid rebound in serum IgM level frequently occurs and can cause symptomatic hyperviscosity. Continuing ibrutinib until the subsequent treatment, with consideration of bridging, may represent a reasonable strategy to maintain disease control. Prospective studies are needed to clarify the optimal management of WM patients with acquired ibrutinib resistance.

\section{Disclosures}

SPT, JJC, GY, and ZRH have received research funding and/or consulting fees from Pharmacyclics and Janssen Pharmaceuticals; SPT has received research funding from Bristol Myers Squibb, X4 Pharmaceuticals, and Beigene; JJC received research funding and/or consulting fees from Abbvie, Beigene, Roche, and TG Therapeutics.

\section{Contributions}

JNG, SS, SPT, and JJC designed the study and performed the data analysis; $M L G, L X, A K, N T, M M, M D, X L, G Y$, and $Z R H$ performed molecular testing on patient samples; $S S, C A F$, $K M, C L, T W, C J P, A R B, S P T$ and JJC took care of the patients and collected the samples; JNG, SPT, and JJC drafted the manuscript. All authors critically reviewed and approved the manuscript.

\section{Funding}

JJC would like to thank the support of the WMR Fund. The authors would also like to thank the Siegel Research Fund for WM, the Orszag Family Fund for WM Research, the D'Amato Family Fund for WM Research, the International Waldenström's Macroglobulinemia Foundation, and the Kerry Robertson Fund for WM. SPT, ZRH and GY are supported by an NIH SPORE in Multiple Myeloma (grant: 2P50CA100707-16A1).

\section{References}

1. Owen RG, Treon SP, Al-Katib A, et al. Clinicopathological definition of Waldenstrom's macroglobulinemia: consensus panel recommendations from the Second International Workshop on Waldenstrom's Macroglobulinemia. Semin Oncol. 2003;30(2):110-115

2. Treon SP, Xu L, Yang G, et al. MYD88 L265P somatic mutation in Waldenström's macroglobulinemia. N Engl J Med. 2012; 367(9):826-833.

3. Hunter ZR, Xu L, Yang G, et al. The genomic landscape of Waldenström macroglobulinemia is characterized by highly recurring MYD88 and WHIM-like CXCR4 mutations, and small somatic deletions associated with B-cell lymphomagenesis. Blood. 2014;123(11):1637-1646.

4. Yang G, Zhou Y, Liu X, et al. A mutation in MYD88 (L265P) supports the survival of lymphoplasmacytic cells by activation of Bruton tyrosine kinase in Waldenström macroglobulinemia. Blood. 2013; 122(7): 1222-1232

5. Yang G, Buhrlage SJ, Tan L, et al. HCK is a survival determinant transactivated by mutated MYD88, and a direct target of ibrutinib. Blood. 2016;127(25):3237-3252.

6.Xu L, Hunter ZR, Tsakmaklis N, et al. Clonal architecture of CXCR4 WHIM-like mutations in Waldenström macroglobulinaemia. Br J Haematol. 2016; 172(5):735744.

7. Cao Y, Hunter ZR, Liu X, et al. The WHIMlike CXCR4S338X somatic mutation activates AKT and ERK, and promotes resistance to ibrutinib and other agents used in the treatment of Waldenstrom's macroglobulinemia. Leukemia. 2015; 29(1): 169-176.

8. Cao Y, Hunter ZR, Liu X, et al. CXCR4 WHIM-like frameshift and nonsense mutations promote ibrutinib resistance but do not supplant MYD88L265P-directed survival signalling in Waldenström macroglobulinaemia cells. Br J Haematol. 2015; 168(5):701-707.

9. Roccaro AM, Sacco A, Jimenez C, et al. C1013G/CXCR4 acts as a driver mutation of tumor progression and modulator of drug resistance in lymphoplasmacytic lymphoma. Blood. 2014;123(26):4120-4131.

10. Treon SP, Tripsas CK, Meid K, et al.
Ibrutinib in previously treated Waldenström's macroglobulinemia. N Engl J Med. 2015;372(15):1430-1440.

11. Treon SP, Meid K, Gustine J, et al. Longterm follow-up of ibrutinib monotherapy in symptomatic, previously treated patients with Waldenström macroglobulinemia. J Clin Oncol. 2021;39(6):565-575.

12. Treon SP, Xu L, Hunter Z. MYD88 mutations and response to ibrutinib in Waldenström's macroglobulinemia. N Engl J Med. 2015;373(6):584-586.

13. Treon SP, Gustine J, Meid K, et al. Ibrutinib monotherapy in symptomatic, treatmentnaïve patients with Waldenström macroglobulinemia. J Clin Oncol. 2018; 36(27):2755-2761.

14. Dimopoulos MA, Trotman J, Tedeschi A, et al. Ibrutinib for patients with rituximabrefractory Waldenstrom's macroglobulinaemia (iNNOVATE): an open-label substudy of an international, multicentre, phase 3 trial. Lancet Oncol. 2017;18(2):241250.

15. Trotman J, Buske C, Tedeschi A, et al. Long-term follow-up of ibrutinib treatment for rituximab-refractory Waldenström's macroglobulinemia: final analysis of the 
Acquired ibrutinib resistance in WM

open-label substudy of the phase 3 iNNOVATETM Trial. Blood. 2020; 136(Suppl 1):S38-39.

16. Tam CS, Opat S, D'Sa S, et al. A randomized phase 3 trial of zanubrutinib vs ibrutinib in symptomatic Waldenström macroglobulinemia: the ASPEN study. Blood. 2020;136(18):2038-2050.

17. Xu L, Tsakmaklis N, Yang G, et al. Acquired mutations associated with ibrutinib resistance in Waldenström macroglobulinemia. Blood. 2017;129(18):2519-2525.

18. Chen JG, Liu X, Munshi M, et al. BTKCys481Ser drives ibrutinib resistance via ERK1/2 and protects BTKwild-type MYD88-mutated cells by a paracrine mechanism. Blood. 2018;131(18):2047-2059.

19. Jiménez C, Chan GG, Xu L, et al. Genomic evolution of ibrutinib-resistant clones in Waldenström macroglobulinaemia. Br J Haematol. 2020;189(6):1165-1170.

20. Gustine JN, Meid K, Dubeau T, et al. Ibrutinib discontinuation in Waldenström macroglobulinemia: etiologies, outcomes, and IgM rebound. Am J Hematol. 2018; 93(4):511-517.

21. Abeykoon JP, Zanwar S, Ansell SM, et al. Ibrutinib monotherapy outside of clinical trial setting in Waldenström macroglobulinaemia: practice patterns, toxicities and outcomes. Br J Haematol. 2020;188(3):394403.

22. Owen RG, Kyle RA, Stone MJ, et al. Response assessment in Waldenström macroglobulinaemia: update from the VIth International Workshop. Br J Haematol. 2013;160(2):171-176.

23. Palladini G, Dispenzieri A, Gertz MA, et al. New criteria for response to treatment in immunoglobulin light chain amyloidosis based on free light chain measurement and cardiac biomarkers: impact on survival outcomes. J Clin Oncol. 2012;30(36):45414549.

24. Cheson BD, Fisher RI, Barrington SF, et al. Recommendations for initial evaluation, staging, and response assessment of Hodgkin and non-Hodgkin lymphoma: the Lugano Classification. J Clin Oncol. 2014; 32(27):3059-3067.

25. Xu L, Hunter ZR, Yang G, et al. MYD88 L265P in Waldenström macroglobulinemia, immunoglobulin $\mathrm{M}$ monoclonal gammopathy, and other B-cell lymphoproliferative disorders using conventional and quantitative allele-specific polymerase chain reaction. Blood. 2013;121(11):2051-2058.

26. Kluk MJ, Lindsley RC, Aster JC, et al. Validation and implementation of a custom next-generation sequencing clinical assay for hematologic malignancies. J Mol Diagn. 2016;18(4):507-515.

27. Treon SP, Ioakimidis L, Soumerai JD, et al. Primary therapy of Waldenström macroglobulinemia with bortezomib, dexamethasone, and rituximab: WMCTG clinical trial 05-180. J Clin Oncol. 2009; 27(23):3830-3835.

28. Dimopoulos MA, García-Sanz R, Gavriatopoulou M, et al. Primary therapy of Waldenstrom macroglobulinemia (WM) with weekly bortezomib, low-dose dexamethasone, and rituximab (BDR): long-term results of a phase 2 study of the European Myeloma Network (EMN). Blood. 2013; 122(19):3276-3282.

29. Rummel MJ, Niederle N, Maschmeyer G, et al. Bendamustine plus rituximab versus $\mathrm{CHOP}$ plus rituximab as first-line treatment for patients with indolent and mantle-cell lymphomas: an open-label, multi- centre, randomised, phase 3 non-inferiority trial. Lancet. 2013;381(9873):1203-1210.

30. Castillo JJ, Gustine JN, Meid K, et al. Response and survival for primary therapy combination regimens and maintenance rituximab in Waldenström macroglobulinaemia. Br J Haematol. 2018;181(1):77-85.

31. Castillo JJ, Gustine JN, Meid K, Dubeau T, Severns P, Treon SP. Ibrutinib withdrawal symptoms in patients with Waldenström macroglobulinemia. Haematologica. 2018; 103(7):e307-e310.

32. Castillo JJ, Gustine JN, Meid K, et al. Impact of ibrutinib dose intensity on patient outcomes in previously treated Waldenström macroglobulinemia. Haematologica. 2018; 103(10):e466-e468.

33. Hodge LS, Ziesmer SC, Yang Z-Z, Secreto FJ, Novak AJ, Ansell SM. Constitutive activation of STAT5A and STAT5B regulates IgM secretion in Waldenstrom's macroglobulinemia. Blood. 2014; 123(7): 1055-1058.

34. Mahajan S, Vassilev A, Sun N, Ozer Z, Mao C, Uckun FM. Transcription factor STAT5A is a substrate of Bruton's tyrosine kinase in B cells. J Biol Chem. 2001;276(33):3121631228.

35. Maddocks KJ, Ruppert AS, Lozanski G, et al. Etiology of ibrutinib therapy discontinuation and outcomes in patients with chronic lymphocytic leukemia. JAMA Oncol. 2015;1(1):80-87.

36. Hampel PJ, Ding W, Call TG, et al. Rapid disease progression following discontinuation of ibrutinib in patients with chronic lymphocytic leukemia treated in routine clinical practice. Leuk Lymphoma. 2019; 60(11):2712-2719.

37. Treon SP. How I treat Waldenström macroglobulinemia. Blood. 2015; 126(6): 721-732.

38. Gustine JN, Meid K, Dubeau T, et al. Serum IgM level as predictor of symptomatic hyperviscosity in patients with Waldenström macroglobulinaemia. Br J Haematol. 2017;177(5):717-725.

39. Treon SP, Branagan AR, Hunter Z, Santos $D$, Tournhilac O, Anderson KC. Paradoxical increases in serum IgM and viscosity levels following rituximab in Waldenstrom's macroglobulinemia. Ann Oncoly. 2004;15(10):1481-1483.

40. Ghobrial IM, Fonseca R, Greipp PR, et al. Initial immunoglobulin $M$ 'flare' after rituximab therapy in patients diagnosed with Waldenstrom macroglobulinemia. Cancer. 2004;101(11):2593-2598.

41. Hampel PJ, Call TG, Ding W, et al. Addition of venetoclax at time of progression in ibrutinib-treated patients with chronic lymphocytic leukemia: Combination therapy to prevent ibrutinib flare. Am J Hematol. 2020;95(3):E57-e60.

42. Castillo JJ, Advani RH, Branagan AR, et al. Consensus treatment recommendations from the tenth International Workshop for Waldenstrom Macroglobulinaemia. Lancet Haematol. 2020;7(11):e827-e837.

43. Castillo J, Allan J, Siddiqi $T$, et al. Multicenter prospective phase II study of venetoclax in patients with previously treated Waldenstrom macroglobulinemia. Clin Lymphoma Myeloma Leuk. 2019;19(10, Supplement):e39-e40.

44. Jones JA, Mato AR, Wierda WG, et al. Venetoclax for chronic lymphocytic leukaemia progressing after ibrutinib: an interim analysis of a multicentre, openlabel, phase 2 trial. Lancet Oncol. 2018; 19(1):65-75.

45. Castillo JJ, Meid K, Gustine JN, et al. Prospective clinical trial of ixazomib, dexamethasone, and rituximab as primary therapy in Waldenström macroglobulinemia. Clin Cancer Res. 2018;24(14):32473252 .

46. Castillo JJ, Meid K, Flynn CA, et al. Ixazomib, dexamethasone, and rituximab in treatment-naive patients with Waldenström macroglobulinemia: longterm follow-up. Blood Adv. 2020; 4(16):3952-3959.

47. Kersten MJ, Minnema MC, Vos JM, et al. Ixazomib, rituximab and dexamethasone (IRD) in patients with relapsed or progressive Waldenstrom's macroblobulinemia: results of the prospective phase I/II HOVON 124/Ecwm-R2 trial. Blood. 2019;134(Suppl 1):S344.

48. Tomowiak C, Desseaux K, Poulain S, et al. Open label non-randomized phase II study exploring "chemo-free " treatment association with idelalisib + obinutuzumab in patients with relapsed/refractory (R/R) Waldenstrom's macroglobulinemia (MW), a Filo trial: results of the intermediary analysis of the induction phase. Blood. 2019;134(Suppl 1):S346.

49. Castillo JJ, Gustine JN, Meid K, et al. Response and survival outcomes to ibrutinib monotherapy for patients with Waldenström macroglobulinemia on and off clinical trials. Hemasphere. 2020; 4(3): 363.

50. Treon SP, Cao Y, Xu L, Yang G, Liu X, Hunter ZR. Somatic mutations in MYD88 and CXCR4 are determinants of clinical presentation and overall survival in Waldenström macroglobulinemia. Blood. 2014;123(18):2791-2796.

51. Castillo JJ, Xu L, Gustine JN, et al. CXCR4 mutation subtypes impact response and survival outcomes in patients with Waldenström macroglobulinaemia treated with ibrutinib. Br J Haematol. 2019; 187(3):356-363

52. Gustine JN, Xu L, Tsakmaklis N, et al. CXCR4S338X clonality is an important determinant of ibrutinib outcomes in patients with Waldenström macroglobulinemia. Blood Adv. 2019;3(19):2800-2803.

53. Buske C, Tedeschi A, Trotman J, et al. Ibrutinib treatment in Waldenström's macroglobulinemia: follow-up efficacy and safety from the iNNOVATE study. Blood. 2018;132(Supplement 1):149.

54. Dimopoulos MA, Tedeschi A, Trotman J, et al. Phase 3 trial of ibrutinib plus rituximab in Waldenström's macroglobulinemia. N Engl J Med. 2018;378(25):2399-2410.

55. Buske C, Tedeschi A, Trotman J, et al. Fiveyear follow-up of Ibrutinib plus rituximab vs. placebo plus rituximab for Waldenstrom's macroglobulinemia: final analysis from the randomized Phase 3 iNNOVATETM Study. Blood. 2020; 136(Supplement 1):24-26.

56. Shinohara $\mathrm{H}$, Inoue $\mathrm{A}$, Toyama-Sorimachi $\mathrm{N}$, et al. Dok-1 and Dok-2 are negative regulators of lipopolysaccharide-induced signaling. J Exp Med. 2005;201(3):333-339.

57. Poulain S, Roumier C, Bertrand E, et al. TP53 mutation and its prognostic significance in Waldenstrom's macroglobulinemia. Clin Cancer Res. 2017;23(20):6325-6335.

58. Gustine JN, Tsakmaklis N, Demos MG, et al. TP53 mutations are associated with mutated MYD88 and CXCR4, and confer an adverse outcome in Waldenström macroglobulinaemia. Br J Haematol. 2019; 184(2):242-245 